Article

\title{
The -173 G/C Polymorphism of the MIF Gene and Inflammatory Bowel Disease Risk: A Meta-Analysis
}

\author{
Yongchun Shen ${ }^{\dagger}$, Shujin Guo ${ }^{\dagger}$, Ting Yang, Liuqun Jia, Lei Chen, Jing An, Tao Wang and \\ Fuqiang Wen *
}

Division of Internal Medicine, West China Hospital of Sichuan University, Chengdu 610041, China; E-Mails: shenyongchun1986@gmail.com (Y.S.); shujinguo@126.com (S.G.); yangting8506@163.com (T.Y.); huaxi198691@126.com (L.J.); resalex@126.com (L.C.); bunnysilent@163.com (J.A.); taowangwest@yahoo.com.cn (T.W.)

$\dagger$ These authors contributed equally to this work.

* Author to whom correspondence should be addressed; E-Mail: wenfuqiang_scu@126.com; Tel.: +86-28-8542-2380; Fax: +86-28-8558-2944.

Received: 15 April 2013 / Accepted: 17 May 2013 / Published: 28 May 2013

\begin{abstract}
The $-173 \mathrm{G} / \mathrm{C}$ polymorphism in the macrophage migration inhibitory factor (MIF) gene has been implicated in susceptibility to inflammatory bowel disease (IBD), but the results are inconclusive. The present meta-analysis aimed to investigate the overall association between the $-173 \mathrm{G} / \mathrm{C}$ polymorphism and IBD risk. We searched in Pubmed, and Embase for studies evaluating the association between the $-173 \mathrm{G} / \mathrm{C}$ gene polymorphism and IBD risk. Data were extracted and statistical analysis was performed using Revman 5.1 and STATA 12.0 software. A total of seven publications involving 4729 subjects (2282 IBD cases and 2447 controls) were included in this meta-analysis. Combined analysis revealed a clear association between this polymorphism and IBD susceptibility (OR $=1.48,95 \% \mathrm{CI}$ : $1.10-2.00, p=0.009$ for CC $v s . \mathrm{CG}+\mathrm{GG}$ ). Subgroup analysis by ethnicity showed that the IBD risk associated with the $-173 \mathrm{G} / \mathrm{C}$ gene polymorphism was significantly elevated among Asians (OR $=1.79,95 \%$ CI: 1.08-2.96, $p=0.02)$, but not among Caucasians. Subgroup analysis by disease suggested that the $-173 \mathrm{G} / \mathrm{C}$ gene polymorphism is a risk factor for ulcerative colitis $(\mathrm{OR}=1.62,95 \% \mathrm{CI}: 1.10-2.37, p=0.01)$, but that it was not associated with Crohn's disease. This meta-analysis suggests that the $-173 \mathrm{G} / \mathrm{C}$ polymorphism in the macrophage MIF gene contributes to IBD susceptibility, specifically in Asian populations. Further studies are needed to validate these findings.
\end{abstract}


Keywords: inflammatory bowel disease; migration inhibitory factor; polymorphism; susceptibility; meta-analysis

\section{Introduction}

Inflammatory bowel disease (IBD), including Crohn's disease (CD) and ulcerative colitis (UC), refers to a heterogeneous group of chronic disorders that leads to cachexia. IBDs are essentially incurable, are of unknown etiology and typically follow a clinical course featuring remissions and relapses [1]. Incidence of IBD is increasing worldwide, and it places a heavy burden on patients because it reduces life quality and ability to work, in addition to increasing disability [2]. The etiology of IBD is complicated and the pathogenesis is poorly understood; nevertheless, growing evidence indicates that IBD results from an abnormal inflammatory response, in which genetic and environmental factors play important roles [3]. Numerous studies have investigated the association of genetic variants with IBD susceptibility [4], and among them, the macrophage migration inhibitory factor (MIF) gene has been highlighted.

MIF is an important pro-inflammatory cytokine that plays a critical role in regulating immune and inflammatory response [5]. Serum MIF levels were found to be significantly higher in patients with UC than in normal controls [6], and another study suggest that immunization with helper $\mathrm{T}$ epitope DNA-vaccine targeting MIF may be a useful approach for the treatment of colitis including inflammatory bowel diseases [7]. These findings suggest a potential role for MIF in IBD pathogenesis and treatment [8]; consistent with this notion, polymorphism in the MIF gene has been associated with susceptibility to inflammatory diseases, such as IBD [9]. Several studies have investigated whether the $-173 \mathrm{G} / \mathrm{C}$ polymorphism in the macrophage MIF gene is associated with IBD risk, and the results have been inconsistent and inconclusive. Since pooled estimates based on meta-analysis have proven to be useful in determining the overall risk of certain IBD polymorphisms when results of individual studies are inconsistent [10], we decided to perform the present meta-analysis in order to clarify the association between the MIF -173G/C polymorphism and IBD risk.

\section{Results and Discussion}

\subsection{Characteristics of Included Studies}

A total of seven publications evaluating the association between the MIF -173G/C polymorphism and IBD risk were included in the meta-analysis, involving 4729 subjects (2282 IBDs cases and 2447 controls) [11-17]. In publications containing both a "CD group" and a "UC group", each group was treated as a separate study in the meta-analysis. Among IBD cases, 1072 were diagnosed with CD and 1210 with UC. One study was excluded because the distribution of genotypes in the control group was inconsistent with HWE [18]. Four of the seven included publications described case-control studies involved Caucasians [12-14,17], while the remaining three publications involving Asians $[11,15,16]$. The characteristics of each case-control study are summarized in Table 1, and genotype and allele distributions for each case-control study are listed in Table 2. 
Table 1. Characteristics of included studies.

\begin{tabular}{|c|c|c|c|c|c|c|c|c|}
\hline Author & Year & Country & Ethnicity & Diagnosis & Cases & Controls & Genotyping method & HWE \\
\hline Dambacher et al.[14] & 2007 & Germany & Caucasian & Crohn's disease & 198 & 159 & PCR & $\mathrm{Y}$ \\
\hline Fei et al. [15] & 2008 & China & Asian & Crohn's disease & 15 & 142 & RFLP-PCR & $\mathrm{Y}$ \\
\hline Fei et al. [15] & 2008 & China & Asian & Ulcerative colitis & 84 & 142 & RFLP-PCR & $\mathrm{Y}$ \\
\hline Griga et al. [12] & 2007 & Germany & Caucasian & Crohn's disease & 157 & 489 & RFLP-PCR & $\mathrm{Y}$ \\
\hline Griga et al. [12] & 2007 & Germany & Caucasian & Ulcerative colitis & 102 & 489 & RFLP-PCR & $\mathrm{Y}$ \\
\hline Nohara et al.[11] & 2004 & Japan & Asian & Ulcerative colitis & 221 & 438 & Tetraprimer-PCR & $\mathrm{Y}$ \\
\hline Oliver et al. [13] & 2007 & Spain & Caucasian & Crohn's disease & 336 & 361 & PCR & $\mathrm{Y}$ \\
\hline Oliver et al. [13] & 2007 & Spain & Caucasian & Ulcerative colitis & 287 & 361 & PCR & $\mathrm{Y}$ \\
\hline Oliver et al. [13] & 2007 & Spain & Caucasian & Crohn's disease & 325 & 526 & PCR & $\mathrm{Y}$ \\
\hline Oliver et al. [13] & 2007 & Spain & Caucasian & Ulcerative colitis & 347 & 526 & PCR & $\mathrm{Y}$ \\
\hline Przybyłowska et al. [17] & 2011 & Poland & Caucasian & Crohn's disease & 41 & 123 & RFLP-PCR & Y \\
\hline Przybyłowska et al. [17] & 2011 & Poland & Caucasian & Ulcerative colitis & 58 & 123 & RFLP-PCR & Y \\
\hline Shiroeda et al.[16] & 2010 & Japan & Asian & Ulcerative colitis & 111 & 209 & SSCP-PCR & $\mathrm{Y}$ \\
\hline
\end{tabular}

Note: PCR: Polymerase chain reaction; RFLP: Restriction fragment length polymorphism; SSCP: Single strand conformation polymorphism; HWE: Hardy-Weinberg equilibrium; Y: Yes.

Table 2. Distribution of migration inhibitory factor (MIF) genotype and allele among inflammatory bowel disease (IBD) patients and controls.

\begin{tabular}{|c|c|c|c|c|c|c|c|c|c|c|}
\hline \multirow{2}{*}{ Author } & \multicolumn{3}{|c|}{ IBD } & \multicolumn{3}{|c|}{ Control } & \multicolumn{2}{|c|}{ IBD } & \multicolumn{2}{|c|}{ Control } \\
\hline & GG & GC & $\mathrm{CC}$ & GG & GC & $\mathbf{C C}$ & $\mathbf{G}$ & $\mathbf{C}$ & $\mathbf{G}$ & $\mathbf{C}$ \\
\hline Dambacher et al. [14] & 146 & 45 & 7 & 108 & 45 & 6 & 335 & 59 & 261 & 57 \\
\hline Fei et al. [15] & 8 & 5 & 2 & 79 & 55 & 8 & 21 & 9 & 213 & 71 \\
\hline Fei et al. [15] & 44 & 27 & 13 & 79 & 55 & 8 & 115 & 53 & 213 & 71 \\
\hline Griga et al. [12] & 116 & 39 & 2 & 318 & 156 & 15 & 271 & 43 & 792 & 186 \\
\hline Griga et al. [12] & 72 & 28 & 2 & 318 & 156 & 15 & 172 & 32 & 792 & 186 \\
\hline Nohara et al.[11] & 135 & 76 & 10 & 288 & 134 & 16 & 346 & 96 & 710 & 166 \\
\hline Oliver et al. [13] & 227 & 94 & 15 & 275 & 81 & 5 & 548 & 124 & 631 & 91 \\
\hline Oliver et al. [13] & 196 & 84 & 7 & 275 & 81 & 5 & 476 & 98 & 631 & 91 \\
\hline Oliver et al. [13] & 239 & 78 & 8 & 406 & 107 & 13 & 556 & 94 & 919 & 133 \\
\hline Oliver et al. [13] & 245 & 87 & 15 & 406 & 107 & 13 & 577 & 117 & 919 & 133 \\
\hline Przybyłowska et al. [17] & 28 & 12 & 1 & 99 & 23 & 1 & 68 & 14 & 221 & 25 \\
\hline Przybyłowska et al. [17] & 38 & 19 & 1 & 99 & 23 & 1 & 95 & 21 & 221 & 25 \\
\hline Shiroeda et al. [16] & 69 & 37 & 5 & 126 & 76 & 7 & 175 & 47 & 328 & 90 \\
\hline
\end{tabular}

\subsection{Quantitative Data Synthesis}

Given the proposal that the recessive model is the best genetic model for evaluating the association between the MIF-173G/C polymorphism in the MIF gene and IBD risk [19], we first analyzed the heterogeneity of CC vs. CG + GG in order to choose the most suitable calculation model. Across all included studies, $\chi^{2}$ was 11.61 and $p=0.48$ for a random-effects model, and $I^{2}$, another index of heterogeneity, was $0 \%$. These findings suggested a lack of heterogeneity. Thus, we chose the fixed-effects model to synthesize the data. The pooled OR based on all studies was 1.48 (95\% CI: 1.10-2.00), which was associated with a $Z$ value of $2.61(p=0.009)$ (Figure 1). This suggested that CC 
homozygotic carriers have a higher risk of IBD than do CG and GG homozygotic individuals. We chose the random-effects model to synthesize the data according to the dominant genetic model. The pooled OR was 1.16 (95\% CI: 0.97-1.39) and the associated $Z$ value was $1.61(p=0.11)$ (Figure 2). These results suggested the possibility that $\mathrm{CC}$ homozygotic carriers and $\mathrm{CG}$ heterozygotic carriers have higher risk of IBD than do GG homozygotic individuals, but the results did not achieve statistical significance. Results for these and other genetic comparisons are summarized in Table 3.

Figure 1. Meta-analysis using a fixed-effects model to evaluate the association between the MIF $-173 \mathrm{G} / \mathrm{C}$ polymorphism and IBD risk (CC vs. CG + GG). The size of the square is proportional to the weight of each study; horizontal lines represent the $95 \%$ CI.

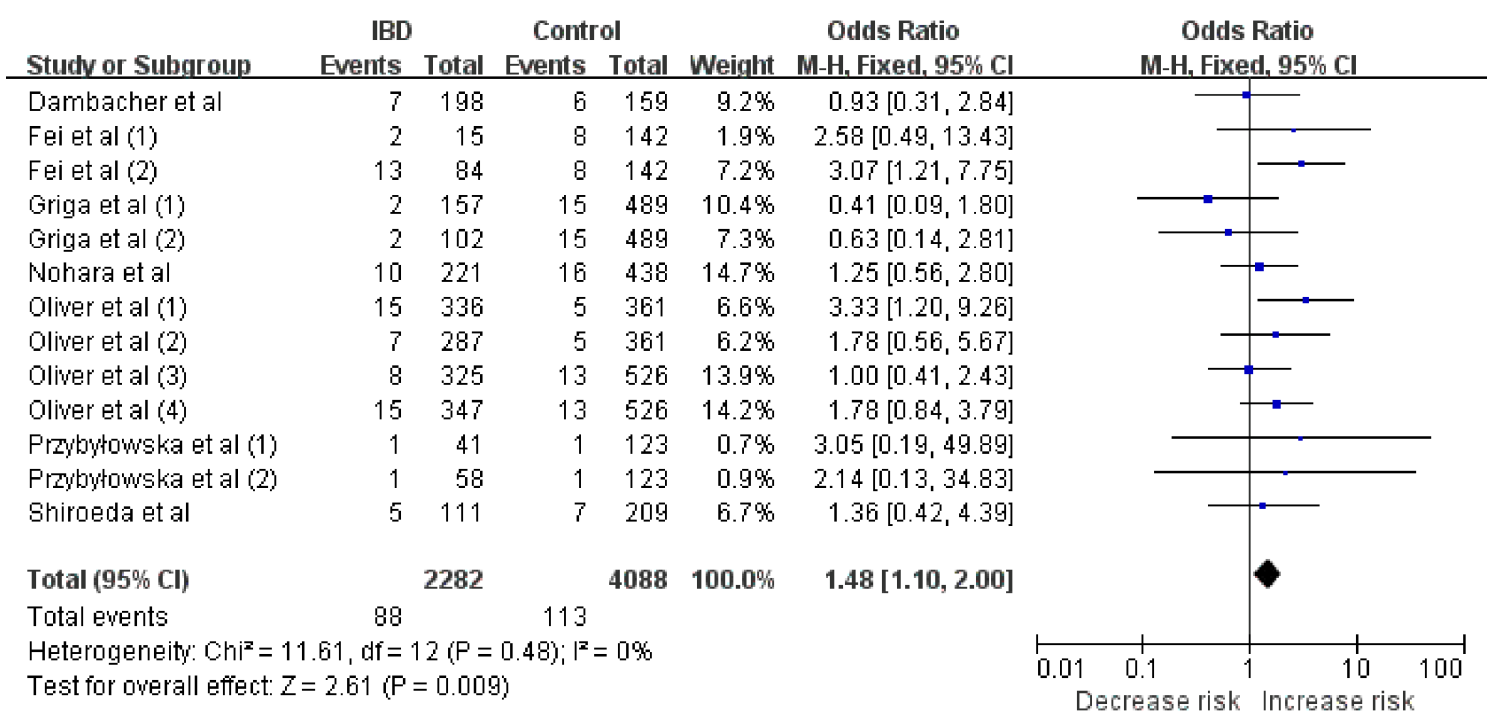

Figure 2. Meta-analysis using a random-effects model to evaluate the association between the MIF $-173 \mathrm{G} / \mathrm{C}$ polymorphism and IBD risk (CC + CG vs. GG). The size of the square is proportional to the weight of each study; horizontal lines represent the $95 \%$ CI.

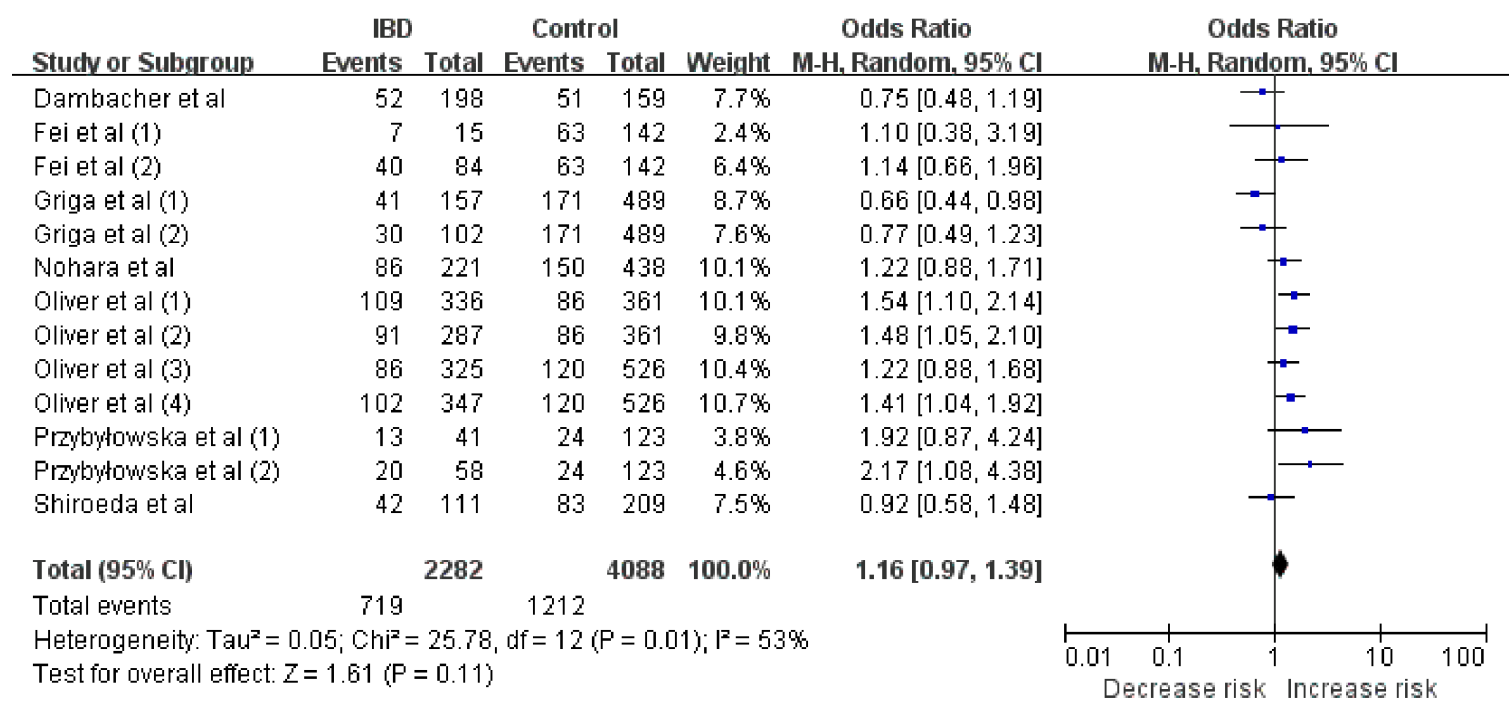


Table 3. Summary of different comparative results.

\begin{tabular}{|c|c|c|c|c|c|c|c|c|}
\hline & \multicolumn{2}{|c|}{ CC + CG vs. GG } & \multicolumn{2}{|c|}{ CC vs. CG + GG } & \multicolumn{2}{|l|}{ CC vs.GG } & \multicolumn{2}{|l|}{ C vs.G } \\
\hline & OR $(95 \% \mathrm{CI})$ & $p^{*}$ & OR $(95 \% \mathrm{CI})$ & $p^{*}$ & OR $(95 \% \mathrm{CI})$ & $p^{*}$ & OR $(95 \% \mathrm{CI})$ & $p^{*}$ \\
\hline Total & $1.16(0.97-1.39)$ & 0.11 & $1.48(1.10-2.00)$ & 0.009 & $1.50(1.12-2.03)$ & 0.007 & $1.18(1.00-1.38)$ & 0.05 \\
\hline \multicolumn{9}{|l|}{ Subgroup by Ethnicity } \\
\hline Caucasian & $1.18(0.93-1.51)$ & 0.17 & $1.35(0.94-1.95)$ & 0.11 & $1.38(0.96-2.00)$ & 0.08 & $1.18(0.94-1.47)$ & 0.16 \\
\hline Asian & $1.12(0.88-1.42)$ & 0.36 & $1.79(1.08-2.96)$ & 0.02 & $1.78(1.06-2.98)$ & 0.03 & $1.18(0.97-1.43)$ & 0.11 \\
\hline \multicolumn{9}{|l|}{ Subgroup by diagnosis } \\
\hline Crohn's disease & $1.08(0.77-1.51)$ & 0.64 & $1.31(0.81-2.09)$ & 0.27 & $1.31(0.82-2.09)$ & 0.27 & $1.11(0.81-1.52)$ & 0.52 \\
\hline Ulcerative colitis & $1.24(1.06-1.44)$ & 0.006 & $1.62(1.10-2.37)$ & 0.01 & $1.65(1.13-2.43)$ & 0.01 & $1.24(1.09-1.41)$ & 0.001 \\
\hline
\end{tabular}

\subsection{Subgroup Analysis}

Subgroup analysis by ethnicity, showed that, among the studies involving Asians [11,15,16], CC homozygotic carriers showed higher risk of IBD than did CG and GG homozygotic individuals, with an OR of 1.79 (95\% CI: 1.08-2.96). Among the studies involving Caucasians [12-14,17], however, no association was found between the MIF $-173 \mathrm{G} / \mathrm{C}$ polymorphism and risk of IBD. When cases with CD or UC were analyzed in separate subgroups, no association was found between the $-173 \mathrm{G} / \mathrm{C}$ polymorphism in the MIF gene and risk of $\mathrm{CD}$, while there was a significant association between the MIF $-173 \mathrm{G} / \mathrm{C}$ polymorphism and risk of UC in all the genetic models. Carriers of the $\mathrm{C}$ allele showed higher risk of UC than did carriers of the G allele, with an OR of 1.24 (95\% CI: $1.09-1.41, p=0.001)$.

\subsection{Sensitivity Analysis and Publication Bias}

To assess the stability of our findings, sensitivity analysis was performed by sequentially excluding each study. Statistically similar results were obtained after sequentially excluding each study, suggesting the stability of the results. Begg's funnel plot and Egger's test were used to assess publication bias. The shape of the funnel plots seemed symmetrical for the CC vs. CG + GG comparison genetic model, suggesting the absence of publication bias (Figure 3). Then, Egger's test was performed to provide statistical evidence of funnel plots asymmetry. The results indicated a lack of publication bias of the present meta-analysis $(p=0.301)$.

Figure 3. Begg's funnel plot to detect publication bias in studies examining the MIF-173G/C polymorphism (CC vs. CG + GG).

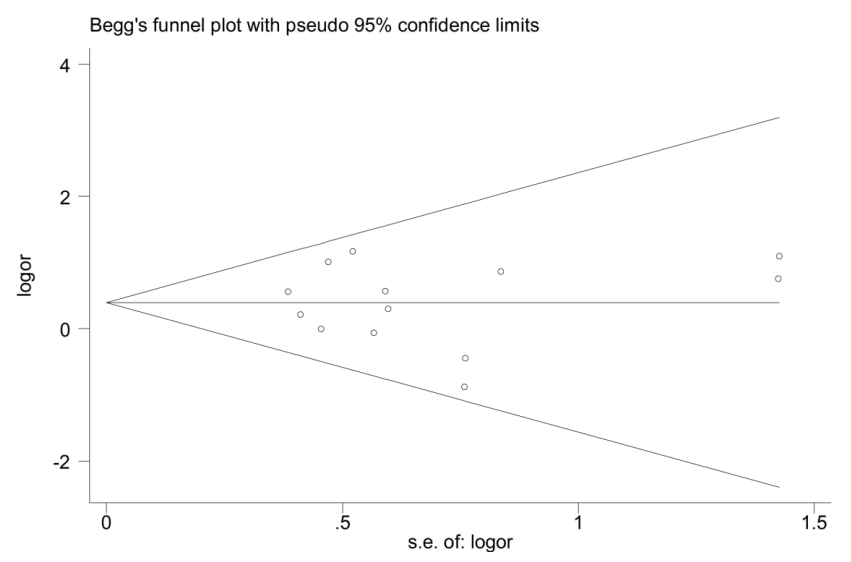




\subsection{Discussion}

IBD refers to a group of chronic relapsing intestinal inflammatory diseases of unidentified causes. Taking epidemiological, genetic and immunological data together, indicates that IBD results from a complex interplay of genetic and environmental factors that produce an intestinal inflammatory response [20,21]. Gene variants may play an important role in the pathogenesis of IBD by altering protein function and individual's susceptibility to disease [2,3,21]. Macrophage MIF has recently been reported to be a key response regulator that acts by directly activating immune cells or by participating in activation pathways initially triggered by other factors [4]. Increasing studies suggest that MIF plays an important role in the regulation of the innate immune response, and MIF may be involved in the pathogenesis of IBD and could be a potential target in the treatment of IBD [8], but studies of the association between the MIF -173G/C polymorphism and risk of IBD have given inconsistent and inconclusive results. Therefore, we performed this meta-analysis to clarify the relationship between this polymorphism and susceptibility to IBD. To our knowledge, it is the first meta-analysis of the possible association between the MIF-173G/C polymorphism and IBD risk.

A total of seven publications describing thirteen case-control studies in Asians and Caucasians were included in this meta-analysis. The effects of dominant/recessive models and of allele frequency were all estimated. In addition, the consistency of genetic effects across different ethnicities and IBD types was investigated. Our findings support the notion that, among Asians, the MIF -173G/C polymorphism plays a role in the development of IBD, and that the homozygotic CC genotype may be a risk factor for IBD.

Our data indicate a strong ethnic bias in the association between the MIF -173G/C polymorphism and risk of IBD. We found the polymorphism to be a risk factor for IBD among Asians, with CC homozygotic carriers at higher risk of IBD than CG and GG homozygotic indivividuals. Among Caucasians, however, we found no evidence of an association between the MIF $-173 \mathrm{G} / \mathrm{C}$ polymorphism and risk of IBD. We also identified a disease-specific bias in the association. While we did not find any evidence of an association between the MIF -173G/C polymorphism and risk of CD, we did find that the polymorphism may be a risk factor for UC, suggesting that the associations between MIF -173G/C polymorphism and IBD risk are ethnicity and disease specific. These findings are consistent with a recent study suggesting that, although the etiology of CD and UC may overlap, the two diseases appear to have different genetic risk profiles [22].

Our finding that the $-173 \mathrm{G} / \mathrm{C}$ polymorphism is a genetic risk factor for IBD in Asians but apparently not Caucasians, suggests population-specific genetic differences in IBD pathogenesis. Therefore, depending on the population, IBD may be associated with different genes, different loci within the same gene, and/or different polymorphisms at the same locus. Indeed, a systematic review of genes associated with susceptibility to IBD in Asian populations found that genetic risk factors differed between Asians and Caucasians [23]. Those authors proposed that new mutations and susceptibility genes identified in Asian IBD patients provide an opportunity to explore new disease-associated mechanisms in this population of rising incidence [23]. Since that review did not examine the macrophage MIF gene, the present meta-analysis extends our appreciation of the complex etiology and genetic risk factors of IBD.

Further research is needed to examine not only the genetic risk factors of IBD, but the environmental risk factors as well. Based on available evidence, we cannot exclude the possibility that environmental risk factors explain at least part of the ethnic bias observed here in the association between the MIF -173G/C 
polymorphism and IBD risk. Therefore further work is essential to tease apart the relative contributions of genes and environment. In addition, to take consideration of population differences will be particularly informative, susceptibility genes identified in IBD patients with different ethnicities provide an opportunity to explore new mechanisms of disease that are specific in different population. What's more, strongly suggesting that blockade of MIF bioactivities by either neutralizing anti-MIF antibodies or antagonists prevents an inflammatory cytokine cascade, which strongly suggests that targeting MIF may be effective for treating IBD [24]. Thus, a comprehensive understanding of genetic, epigenetic, environmental, and clinical factors may not only improve our understanding of the mechanisms of IBD, but also lead to more effective prevention and treatment [25].

The findings in this meta-analysis should be interpreted with caution because of several limitations. First, a relatively small number of studies and subjects were included in this meta-analysis, which may reduce the statistical power for identifying possible associations between the MIF -173G/C polymorphism and IBD risk. In particular, the apparent lack of association between this polymorphism and disease risk in Caucasians and in patients with CD should be verified in larger-scale studies. Second, the included publications were limited to Asian and Caucasian populations, so future work should examine other populations, such as Latinos, especially given substantial evidence of ethnic bias in the $-173 \mathrm{G} / \mathrm{C}$ polymorphism. Third, although we did not set any language restrictions during our literature searching, we included only English-language publications in the meta-analysis. It is possible that our results would be different if they included the findings of unpublished studies or of relevant studies published in other languages.

\section{Experimental Section}

\subsection{Literature Search}

Two authors independently performed systematic searches of Pubmed and Embase databases in January 2013 to identify studies examining the association between the $-173 \mathrm{G} / \mathrm{C}$ polymorphism in the MIF gene and IBD risk. Search terms were as follows: "IBD or inflammatory bowel disease or Crohn's disease or ulcerative colitis" in combination with "migration inhibitory factor" in combination with "polymorphism or variant or mutation". The reference lists of identified studies and review articles were manually searched to find additional relevant publications.

\subsection{Study Selection}

Studies were included in the meta-analysis if they satisfied the following inclusion criteria: (1) they evaluated the potential association between the MIF gene-173G/C polymorphism and IBD risk; (2) they were case-control studies; (3) genotype distributions were available for cases and controls in order to estimate an odds ratio (OR) with $95 \%$ confidence interval (CI); (4) the distribution of genotypes in the control group was consistent with Hardy-Weinberg equilibrium (HWE). Abstracts, reviews, and studies in which genotype frequencies were not reported were excluded; and (5) when publications involved the same or overlapping data sets, only the study with the largest number of participants was included. 


\subsection{Data Extraction}

Two reviewers independently extracted data from the final set of included studies. The following data were extracted: the name of the first author, year of publication, country of origin, ethnicity, sample size, IBD diagnosis, genotyping method, and genotype frequencies in IBD cases and controls.

\subsection{Statistical Analysis}

The strength of the association between the MIF -173G/C polymorphism and risk of IBD was assessed using ORs and 95\% CIs. The significance of the pooled OR was determined using the Z-test and $p<0.05$ was considered statistically significant. First, we evaluated the dominant model $(\mathrm{CC}+\mathrm{CG}$ vs. GG) and recessive model (CC vs. CG + GG), followed by the additive model (CC vs. GG). We also estimated the association based on allelic contrast (C vs. G). To evaluate whether the association showed any ethnicity- or disease-specific effects, we analyzed the data for separate subgroups defined by ethnicity and diagnosis with CD or UC.

Heterogeneity was evaluated using a $\chi^{2}$-based Q statistic and $I^{2}$ statistic, with $p<0.10$ considered statistically significant. When $p \geq 0.10$, the pooled OR of each study was calculated using a fixed-effects model; otherwise, a random-effects model was used.

Publication bias was assessed using Begg's funnel plots and Egger's test [26,27]. Sensitivity analysis was performed by sequentially excluding individual studies and recalculating the results. Pearson's $\times 2$ test was used to determine whether the observed frequencies of genotypes in control group conformed to the HWE[28]. All statistical tests were performed using Revman 5.1 and STATA 12.0 software.

\section{Conclusions}

To the best of our knowledge, this is the first meta-analysis to assess the relationship between the MIF-173G/C polymorphism and IBD risk. Our results suggest that the $-173 \mathrm{G} / \mathrm{C}$ polymorphism is a risk factor for IBD in Asians but not in Caucasians. Large well-designed, multi-center epidemiological studies should be carried out in these and other ethnic populations to confirm our findings.

\section{Acknowledgments}

This work was supported by grants from the National Natural Science Foundation of China (\#81230001 and 31171103 to Fuqiang Wen, \# 31000513 to Tao Wang).

We are indebted to the authors of the primary studies included in this meta-analysis; without their contributions, this work would not have been possible.

\section{Conflict of Interest}

The authors declare no conflict of interest.

\section{References}

1. Cosnes, J.; Gower-Rousseau, C.; Seksik, P.; Cortot A. Epidemiology and natural history of inflammatory bowel diseases. Gastroenterology 2011, 140, 1785-1794. 
2. Stone, C.D. The economic burden of inflammatory bowel disease: Clear problem, unclear solution. Dig. Dis. Sci. 2012, 57, 3042-3044.

3. Cho, J.H.; Brant, S.R. Recent insights into the genetics of inflammatory bowel disease. Gastroenterology 2011, 140, 1704-1712.

4. Neuman, M.G.; Nanau, R.M. Single-nucleotide polymorphisms in inflammatory bowel disease. Transl. Res. 2012, 160, 45-64.

5. Hoi, A.Y.; Iskander, M.N.; Morand, E.F. Macrophage migration inhibitory factor: A therapeutic target across inflammatory diseases. Inflamm. Allergy Drug Targets. 2007, 6, 183-273.

6. Murakami, H.; Akbar, S.M.; Matsui, H.; Onji, M. Macrophage migration inhibitory factor in the sera and at the colonic mucosa in patients with ulcerative colitis: Clinical implications and pathogenic significance. Eur. J. Clin. Invest. 2001, 31, 337-343.

7. Ohkawara, T.; Koyama, Y.; Onodera, S.; Takeda, H.; Kato, M.; Asaka, M.; Nishihira, J. DNA vaccination targeting macrophage migration inhibitory factor prevents murine experimental colitis. Clin. Exp. Immunol. 2011, 163, 113-122.

8. Nishihira, J.; Mitsuyama, K. Overview of the role of macrophage migration inhibitory factor (MIF) in inflammatory bowel disease. Curr. Pharm. Des. 2009, 15, 2104-2109.

9. Renner, P.; Roger, T.; Calandra, T. Macrophage migration inhibitory factor: Gene polymorphisms and susceptibility to inflammatory diseases. Clin. Infect. Dis. 2005, 41, S513-S519.

10. Nakaoka, H.; Inoue, I. Meta-analysis of genetic association studies: Methodologies, between-study heterogeneity and winner's curse. J. Hum. Genet. 2009, 54, 615-623.

11. Nohara, H.; Okayama, N.; Inoue, N.; Koike, Y.; Fujimura, K.; Suehiro, Y.; Hamanaka, Y.; Higaki, S.; Yanai, H.; Yoshida, T.; et al. Association of the $-173 \mathrm{G} / \mathrm{C}$ polymorphism of the macrophage migration inhibitory factor gene with ulcerative colitis. J. Gastroenterol. 2004, 39, 242-246.

12. Griga, T.; Wilkens, C.; Wirkus, N.; Epplen, J.; Schmiegel, W.; Klein, W. A polymorphism in the macrophage migration inhibitory factor gene is involved in the genetic predisposition of Crohn's disease and associated with cumulative steroid doses. Hepatogastroenterology 2007, 54, 784-786.

13. Oliver, J.; Márquez, A.; Gómez-Garcia, M.; Martinez, A.; Mendoza, J.L.; Vilchez, J.R.; López-Nevot, M.A.; Piñero, A.; de la Concha, E.G.; Nieto, A.; et al. Association of the macrophage migration inhibitory factor gene polymorphisms with inflammatory bowel disease. Gut 2007, 56, 150-151.

14. Dambacher, J.; Staudinger, T.; Seiderer, J.; Sisic, Z.; Schnitzler, F.; Pfennig, S.; Hofbauer, K.; Konrad, A.; Tillack, C.; Otte, J.M.; et al. Macrophage migration inhibitory factor (MIF) -173G/C promoter polymorphism influences upper gastrointestinal tract involvement and disease activity in patients with Crohn's disease. Inflamm. Bowel. Dis. 2007, 13, 71-82.

15. Fei, B.Y.; Lv, H.X.; Yang, J.M.; Ye, Z.Y. Association of MIF-173 gene polymorphism with inflammatory bowel disease in Chinese Han population. Cytokine 2008, 41, 44-51.

16. Shiroeda, H.; Tahara, T.; Nakamura, M.; Shibata, T.; Nomura, T.; Yamada, H.; Hayashi, R.; Saito, T.; Yamada, M.; Fukuyama, T.; et al. Association between functional promoter polymorphisms of macrophage migration inhibitory factor (MIF) gene and ulcerative colitis in Japan. Cytokine 2010, $51,173-177$. 
17. Przybyłowska, K.; Mrowicki, J.; Sygut, A.; Narbutt, P.; Dziki, Ł.; Dziki, A.; Majsterek, I. Contribution of the $-173 \mathrm{G} / \mathrm{C}$ polymorphism of macrophage migration inhibitory factor gene to the risk of inflammatory bowel diseases. Pol. PrzeglChir. 2011, 83, 76-80.

18. Sivaram, G.; Tiwari, S.K.; Bardia, A.; Anjum, F.; Vishnupriya, S.; Habeeb, A.; Khan, A.A. Macrophage migration inhibitory factor, Toll-like receptor 4, and CD14 polymorphisms with altered expression levels in patients with ulcerative colitis. Hum. Immunol. 2012, 73, 201-205.

19. Thakkinstian, A.; McElduff, P.; D’Este, C.; Duffy, D.; Attia, J. A method for meta-analysis of molecular association studies. Stat. Med. 2005, 24, 1291-1306.

20. Latella, G.; Papi, C. Crucial steps in the natural history of inflammatory bowel disease. World J. Gastroenterol. 2012, 18, 3790-3799.

21. Tsianos, E.V.; Katsanos, K.H.; Tsianos, V.E. Role of genetics in the diagnosis and prognosis of Crohn's disease. World J. Gastroenterol. 2012, 18, 105-118.

22. Nakagome, S.; Takeyama, Y.; Mano, S.; Sakisaka, S.; Matsui, T.; Kawamura, S.; Oota, H. Population-specific susceptibility to Crohn's disease and ulcerative colitis; dominant and recessive relative risks in the Japanese population. Ann. Hum. Genet. 2010, 74, 126-136.

23. Ng, S.C.; Tsoi, K.K.; Kamm, M.A.; Xia, B.; Wu, J.; Chan, F.K.; Sung, J.J. Genetics of inflammatory bowel disease in Asia: Systematic review and meta-analysis. Inflamm. Bowel. Dis. 2012, 18, 1164-1176.

24. Nishihira, J. Molecular function of macrophage migration inhibitory factor and a novel therapy for inflammatory bowel disease. Ann. N. Y. Acad. Sci. 2012, 1271, 53-57.

25. Cheon, J.H. Genetics of inflammatory bowel diseases: A comparison between western and eastern perspectives. J. Gastroenterol. Hepatol. 2012, 28, 220-226.

26. Begg, B.C.; Mazumdar, M. Operating characteristics of a rank correlation test for publication bias. Biometrics 1994, 50, 1088-1101.

27. Egger, M.; Davey Smith, G.; Schneider, M.; Minder, C. Bias in meta-analysis detected by a simple, graphical test. BMJ 1997, 315, 629-634.

28. Schaid, D.J.; Jacobsen, S.J. Biased tests of association: Comparisons of allele frequencies when departing from Hardy-Weinberg proportions. Am. J. Epidemiol. 1999, 149, 706-711.

(C) 2013 by the authors; licensee MDPI, Basel, Switzerland. This article is an open access article distributed under the terms and conditions of the Creative Commons Attribution license (http://creativecommons.org/licenses/by/3.0/). 